\title{
Lateral-Apical Approach to Pericardiocentesis for Treatment of Cardiac Tamponade Immediately Post-orthotopic Liver Transplantation
}

\author{
Christan D. Santos $^{1}$, Joshua A. Propst ${ }^{2}$, Juan M. Canabal ${ }^{2}$, Rohan M. Goswami ${ }^{2}$ \\ 1. Department of Critical Care Medicine, Mayo Clinic, Jacksonville, USA 2. Department of Transplant Medicine, Mayo \\ Clinic, Jacksonville, USA
}

Corresponding author: Christan D. Santos, santos.christan@mayo.edu

\begin{abstract}
Cardiac tamponade is a rare complication following orthotopic liver transplantation (OLT). The incidence and treatment specific to the immediate postoperative OLT patient have never been reported. Here, we describe a case of OLT complicated by coagulopathy and difficult intraoperative pulmonary artery catheter placement with subsequent postoperative hemopericardium resulting in tamponade. An emergent, ultrasound-guided, lateral-apical pericardiocentesis was successfully performed, suggesting a possible procedural technique for pericardiocentesis in the immediate postoperative period for liver transplant patients.
\end{abstract}

Review began 04/15/2021 Review ended 06/10/2021 Published 06/16/2021

๑) Copyright 2021

Santos et al. This is an open access article distributed under the terms of the Creative Commons Attribution License CC-BY 4.0., which permits unrestricted use, distribution, and reproduction in any medium, provided the original author and source are credited.
Categories: Anesthesiology, Cardiac/Thoracic/Vascular Surgery, Transplantation

Keywords: tamponade, liver transplant, orthotopic liver transplant, pericardiocentesis, cardiac tamponade, point-ofcare ultrasound

\section{Introduction}

Cardiac tamponade following orthotopic liver transplantation (OLT) is an uncommon occurrence that is rarely reported in the literature. Cardiac chamber perforation secondary to pulmonary artery catheter (PAC) insertion is also rare [1-4]. Cardiac tamponade should be considered and confirmed quickly with point-ofcare ultrasound (POCUS) in patients with acute cardiogenic shock following either one of these procedures as delay in recognition and management can have dire consequences [5]. Bedside pericardiocentesis was historically performed via the subxiphoid approach. However, ultrasound-guided pericardiocentesis (UGP) has been performed since 1979 and has been widely documented to be safe and efficacious for the management of cardiac tamponade [6]. Here, we present a case of cardiac tamponade post-OLT with successful UGP via a lateral-apical approach.

This article was previously presented as a meeting abstract (abstract number 1702) at the 2020 Society of Critical Care Medicine, 49th Critical Care Congress on February 16, 2020, at the Orange County Convention Center in Orlando, Florida, United States of America.

\section{Case Presentation}

A 70-year-old female with renal insufficiency, Crohn's disease, and alcoholic cirrhosis complicated by ascites, hepatic encephalopathy, and coagulopathy underwent OLT with intraoperative hemodynamic and echocardiographic monitoring via PAC and transesophageal echocardiography. Intraoperative complications included coagulopathy requiring multiple transfusions and difficult PAC placement requiring multiple insertion attempts and readjustments. Postoperatively, the patient was transferred to the intensive care unit (ICU) in stable condition without vasoactive support.

Within an hour of arrival, she became tachycardic (110-120 beats per minute) and severely hypotensive (mean arterial pressure of $40-50 \mathrm{mmHg}$ ) unresponsive to volume resuscitation. Central venous pressures (CVP) acutely rose to greater than $20 \mathrm{mmHg}$ with near equalization of CVP (20-25 mmHg) and diastolic pulmonary artery pressure (25-30 $\mathrm{mmHg})$. Phenylephrine and epinephrine boluses were attempted without improvement in her hemodynamics. Cardiopulmonary resuscitation was not initiated nor indicated during this period. POCUS revealed a large pericardial effusion with biatrial and biventricular collapse compatible with tamponade physiology (Figure 1). 


\section{Cureus}

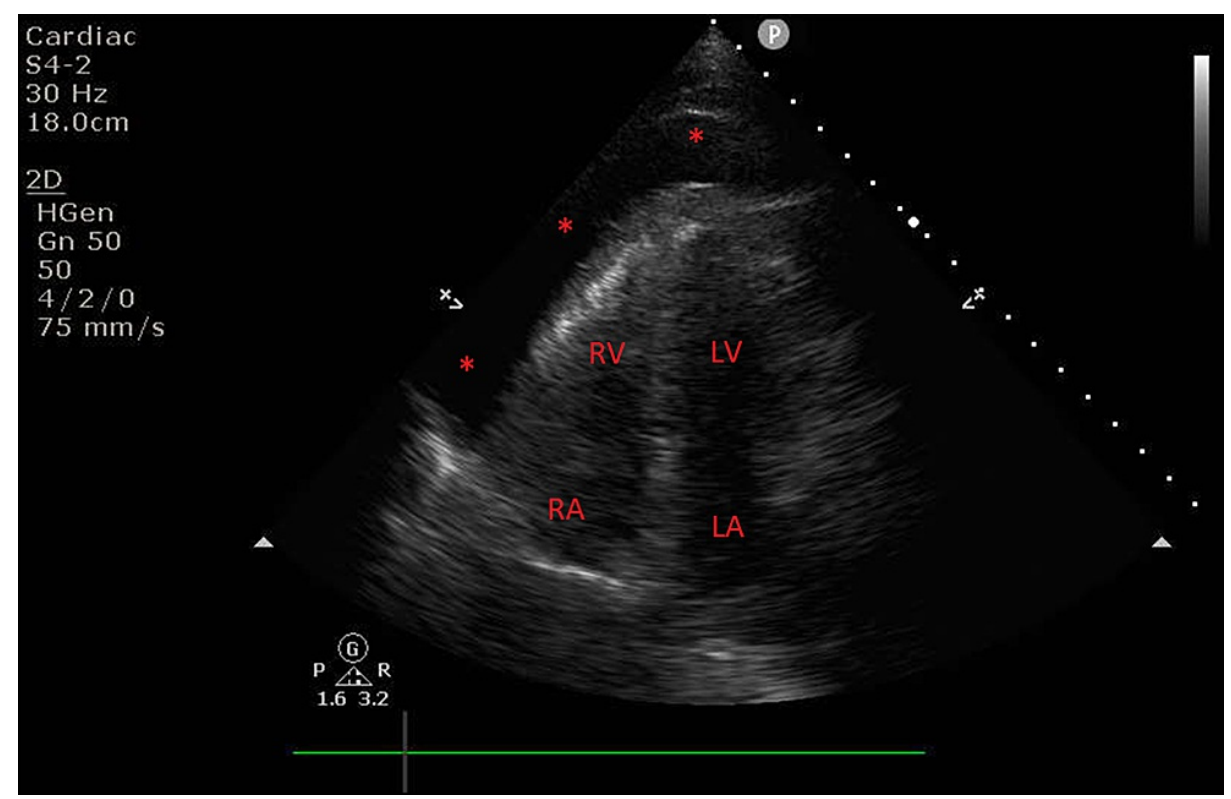

\section{FIGURE 1: Large pericardial effusion.}

Transthoracic apical echocardiographic view revealing large pericardial effusion as noted by red asterisks.

RA: right atrium; RV: right ventricle; LA: left atrium; LV: left ventricle

Color Doppler revealed a small area of diastolic flow exiting the right atrial (RA) free wall at the RA-inferior vena cava junction concerning for perforation (Figure 2).

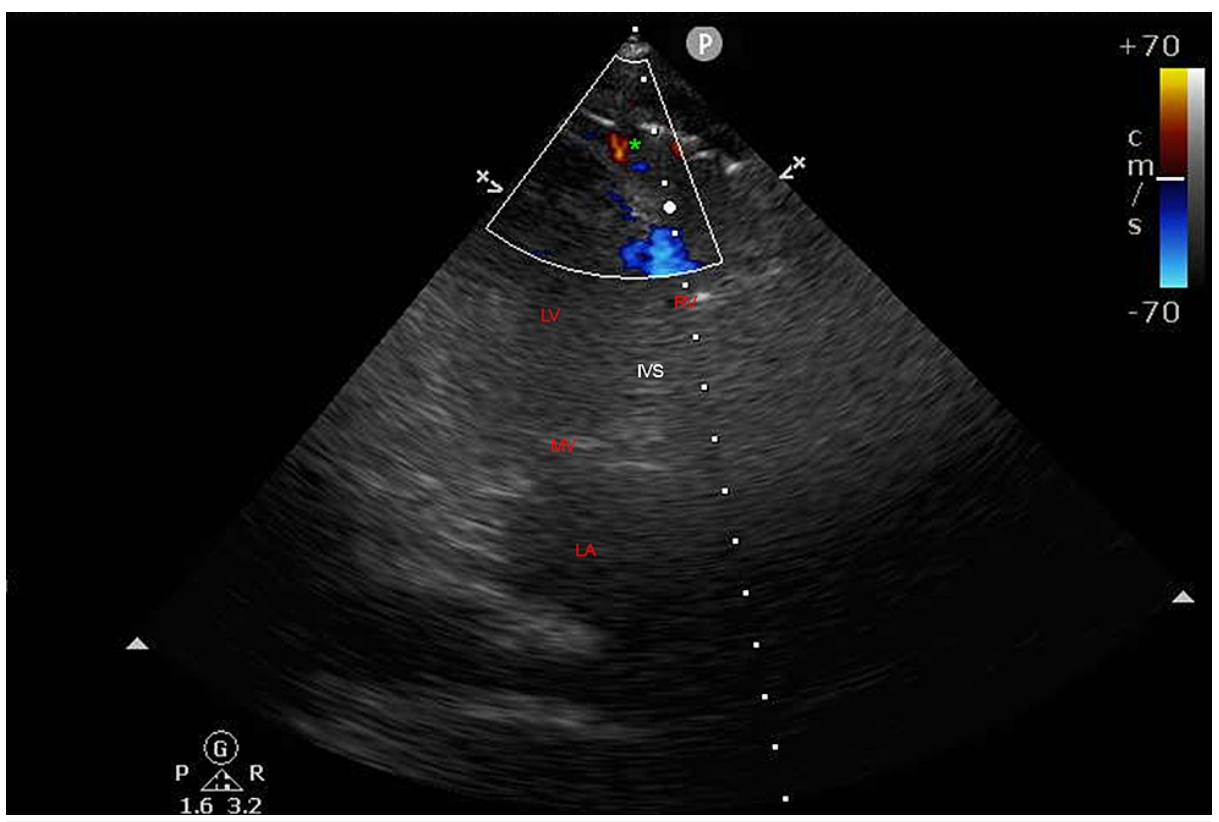

FIGURE 2: Color Doppler revealing extracardiac flow.

Transthoracic echocardiographic view of extracardiac flow as indicated by green asterisk.

RV: right ventricle; LA: left atrium; LV: left ventricle; MV: mitral valve; IVS: interventricular septum

Emergent bedside UGP was performed with a lateral-apical approach, as a subxiphoid approach was unavailable due to the size and position of the left lobe of the graft. An 8-French pigtail drainage catheter was placed with immediate return of $350 \mathrm{~mL}$ of frank blood and normalization of hemodynamics. Due to acute blood loss, anemia, and deranged thromboelastogram, the patient received two units of red blood 
cells, 20 units of cryoprecipitate, one unit of platelets, and desmopressin $(0.3 \mathrm{mcg} / \mathrm{kg})$. Given the patient's stability, and after consultation with cardiothoracic surgery, invasive repair was deferred. Serial POCUS revealed stable biventricular function without reaccumulation of pericardial fluid. The drain was removed 48 hours after placement. The patient transferred out of the ICU on postoperative day (POD) three and was discharged home on POD eight. She was followed in the outpatient setting weekly for the month following discharge. She was noted to have normal allograft function, stable renal insufficiency, and no further cardiac complications.

\section{Discussion}

Cardiac tamponade occurs when intrapericardial pressure is elevated due to air or fluid collections which constrain cardiac filling causing life-threatening hemodynamic instability. It is primarily a clinical diagnosis with a constellation of hemodynamic and echocardiographic changes [7]. Cardiac tamponade is rare in OLT patients; however, their inherent thrombocytopenia and coagulopathy increase the risk of hemopericardium in the setting of atrial perforation, as seen in this case.

Invasive hemodynamic monitoring via PAC insertion during liver transplantation varies across facilities. Traditional PACs measured cardiac output by intermittent thermodilution which was limited in accuracy by several user-dependent techniques as well as administration of large volumes of intravenous fluids and cooled blood products during transplantation. The new generation of PACs utilize heat rather than cold thermodilution via a thermal filament connected to the catheter, resulting in continuous cardiac output monitoring [8]. Improvements in algorithms and computational techniques have resulted in the calculation of global end-diastolic volume and the right ventricular end-diastolic and end-systolic volumes, all of which provide a more accurate estimation of intravascular blood volume [9]. Our facility routinely utilizes PACs for hemodynamic monitoring and intravascular volume assessment during liver transplantation. Some facilities reserve this resource only for patients with portopulmonary hypertension, as these patients have been found to have high perioperative mortality [9].

Chamber perforation secondary to PAC insertion is rare, as low as $0.011 \%$ according to one clinical audit of 9,071 insertions over 16 years [1-4]. It is difficult to discern if perforation is caused by the guidewire or the catheter itself. However, with routine use of curved tip guidewires, the likelihood of injury caused by the wire is low. In this patient, it is most likely that the perforation occurred during the passage of the PAC from the RA to the right ventricle, perhaps secondary to balloon under-inflation. Delay in symptomology until the postoperative period is likely due to discontinuation of active coagulopathy correction.

Procedural ultrasound guidance has become the standard of care when performing pericardiocentesis as treatment of cardiac tamponade. It has been found to be safe, effective, and easy to perform, with minimal complications and increased success rates, even in patients with coagulopathy and thrombocytopenia $[6,10]$. Typical approaches include subxiphoid, apical, and parasternal. However, the apical approach is typically avoided due to a higher risk of ventricular puncture [6]. Utilization of POCUS has been found to provide rapid, noninvasive differentiation of shock as well as reduce procedural complications and improve patient safety [11-13].

\section{Conclusions}

In our patient, a lateral-apical approach to UGP was utilized to avoid puncturing the transplanted allograft. The coagulopathic nature of any liver failure patient inherently increases hemorrhagic complications of invasive procedures. With echocardiographic guidance, this patient's hemopericardium was drained without complication and hemodynamic stability was restored. This case demonstrates patient-specific adaptation of an emergent bedside procedure made possible by ultrasound, further demonstrating the need for POCUS competency among all intensive care providers.

\section{Additional Information \\ Disclosures}

Human subjects: Consent was obtained or waived by all participants in this study. Conflicts of interest: In compliance with the ICMJE uniform disclosure form, all authors declare the following: Payment/services info: All authors have declared that no financial support was received from any organization for the submitted work. Financial relationships: All authors have declared that they have no financial relationships at present or within the previous three years with any organizations that might have an interest in the submitted work. Other relationships: All authors have declared that there are no other relationships or activities that could appear to have influenced the submitted work.

\section{References}

1. Procaccini B, Clementi G: [Pulmonary artery catheterization in 9071 cardiac surgery patients: a review of complications]. Ital Heart J Suppl. 2004, 5:891-9.

2. Hadian M, Pinsky MR: Evidence-based review of the use of the pulmonary artery catheter: impact data and complications. Crit Care. 2006, 10 Suppl 3:S8. 10.1186/cc4834 
3. Harvey S, Harrison DA, Singer M, et al.: Assessment of the clinical effectiveness of pulmonary artery catheters in management of patients in intensive care (PAC-Man): a randomized controlled trial. Lancet. 2005, 366:472-7. 10.1016/S0140-6736(05)67061-4

4. Lu SY, Matsusaki T, Abuelkasem E, et al.: Complications related to invasive hemodynamic monitors during adult liver transplantation. Clin Transplant. 2013, 27:823-8. 10.1111/ctr.12222

5. Tsang TS, Oh JK, Seward JB: Diagnosis and management of cardiac tamponade in the era of echocardiography. Clin Cardiol. 1999, 22:446-52. 10.1002/clc.4960220703

6. Maggiolini S, De Carlini CC, Imazio M: Evolution of the pericardiocentesis technique . J Cardiovasc Med (Hagerstown). 2018, 19:267-73. 10.2459/JCM.0000000000000649

7. Kearns MJ, Walley KR: Tamponade: hemodynamic and echocardiographic diagnosis. Chest. 2018, 153:126675. 10.1016/j.chest.2017.11.003

8. Rudnick MR, Marchi LD, Plotkin JS: Hemodynamic monitoring during liver transplantation: a state of the art review. World J Hepatol. 2015, 7:1302-11. 10.4254/wjh.v7.i10.1302

9. Singh S, Nasa V, Tandon M: Perioperative monitoring in liver transplant patients . J Clin Exp Hepatol. 2012, 2:271-8. 10.1016/j.jceh.2012.06.003

10. Ryu AJ, Kane GC, Pislaru SV, et al.: Bleeding complications of ultrasound-guided pericardiocentesis in the presence of coagulopathy or thrombocytopenia. J Am Soc Echocardiogr. 2020, 33:399-401. 10.1016/j.echo.2019.11.006

11. Milne J, Atkinson P, Lewis D, et al.: Sonography in hypotension and cardiac arrest (SHoC): rates of abnormal findings in undifferentiated hypotension and during cardiac arrest as a basis for consensus on a hierarchical point of care ultrasound protocol. Cureus. 2016, 8:e564. 10.7759/cureus.564

12. Brass P, Hellmich M, Kolodziej L, Schick G, Smith AF: Ultrasound guidance versus anatomical landmarks for internal jugular vein catheterization. Cochrane Database Syst Rev. 2015, 1:CD006962. 10.1002/14651858.CD006962.pub2

13. Bhagra A, Tierney DM, Sekiguchi H, Soni NJ: Point-of-care ultrasonography for primary care physicians and general internists. Mayo Clin Proc. 2016, 91:1811-27. 10.1016/j.mayocp.2016.08.023 